\title{
Extraoccipital benign childhood partial seizures with ictal vomiting and excellent prognosis
}

\author{
C P Panayiotopoulos
}

\begin{abstract}
In a previous report of 900 patients with epileptic seizures, 24 children had ictal vomiting. Twelve had a previously unrecognised syndrome of early onset benign childhood occipital seizures (EBOS) and three had symptomatic epilepsy. The other nine children with extraoccipital EEG foci or normal EEG are described in this paper based on a prospective follow up for a median of 9 years after their first seizure. All had normal neurology, mental state, and development. All seizures of all but one patient occurred in sleep. Seizures manifested with ictal vomiting (nine), deviation of the eyes (four), speech arrest (three), hemiconvulsions (five), oropharyngolaryngeal symptoms, and hypersalivation (one) with or without impairment of consciousness. Median age at onset was 5 years, seizures were infrequent and remitted at a median age of 6. Four children had a single fit, four had two to three, and only one child had many seizures before the initiation of treatment. This study certifies that idiopathic childhood partial seizures with ictal vomiting may occur with EEG spike foci in other than the occipital regions or EEG may be normal. Despite some clinico-EEG differences from the EBOS, childhood seizures with ictal vomiting, and extraoccipital EEG foci are of equally excellent prognosis. Their existence on the border between rolandic and occipital seizures is consistent with a unified concept of a benign childhood partial seizure susceptibility syndrome.

(F Neurol Neurosurg Psychiatry 1999;66:82-85)
\end{abstract}

Keywords: benign childhood partial seizures; prognosis; EEG spike foci

Department of Clinical Neurophysiology and Epilepsies, St Thomas' Hospital, London SE1 7EH, UK. Telephone 00441719228221 ; fax 00441719228263

C P Panayiotopoulos

Received 6 April 1998 and in final form

29 July 1998

Accepted 10 August 1998 otopoulos in a 15 year prospective study ${ }^{1}$ is now well established ${ }^{2-6}$ as the most frequent after the centrotemporal (rolandic) phenotype of benign childhood partial seizures (BCPS). ${ }^{7}$

Ictal vomiting, often with deviation of the eyes, is a main symptom of EBOS that are usually nocturnal and long, often progressing to hemiconvulsions or generalised convulsions.
Peak age at onset is 5 years, seizures are singular or infrequent and remit within 1-2 years. The likelihood of developing seizures after the age of 12 is less than of febrile convulsions. The EEG shows occipital paroxysms or occipital spikes. Spike foci in other locations may occur with or after the disappearance of occipital spikes and, rarely, patients may later develop other forms of BCPS or idiopathic occipital photosensitivity. ${ }^{1-9}$

Thus ictal vomiting, previously considered as extremely rare ${ }^{10}$ or dismissed as a non-ictal or a migraine symptom, is the commonest manifestation of EBOS. ${ }^{6}$

In another study ${ }^{11}$ of 900 seizure patients of all ages, ictal vomiting occurred in 24 children. Twelve had EBOS and three symptomatic partial seizures. The remaining nine idiopathic patients had EEG spike foci in other than the occipital regions (five children), a photoparoxysmal response (one), or normal EEG (three). The purpose of this report is to describe the clinical manifestations and output of a long follow up of these nine children. By virtue of an excellent prognosis, they classify among the benign childhood partial seizures.

Methods and patients

These have been previously detailed. ${ }^{11}$ Of 900 patients with epileptic seizures, 418 had seizure onset before the age of 13 years. Ninety four had BCPS classified according to the main EEG spike localisation ${ }^{72}$ into $72(76.6 \%)$ with centrotemporal spikes, 18 (17\%) with occipital paroxysms, two with frontal and two with midline spikes. ${ }^{1}$ Ictal vomiting occurred in only 24 children. ${ }^{11}$ Postictal vomiting was excluded.

PROSPECTIVE STUDY

All patients with occipital seizures, EEG occipital spikes, or both were prospectively followed up from $1973 .^{1}$ Eighteen patients with benign childhood occipital seizures were reported in detail. ${ }^{1}$
PATIENTS OF THIS REPORT

All nine patients of normal neurological state and development with normal brain scan had ictal vomiting either from the onset or during the ictus. 
Table Chronological, clinical, ictal, and EEG data

\begin{tabular}{|c|c|c|c|c|c|c|c|c|c|}
\hline \multirow[b]{2}{*}{$\begin{array}{l}\text { Case } \\
\text { No/Sex }\end{array}$} & \multicolumn{3}{|c|}{ Age (y) at } & \multicolumn{5}{|l|}{ Seizures } & \multirow[b]{2}{*}{ EEG localisation } \\
\hline & Last FU & Onset & $\begin{array}{l}\text { Last } \\
\text { Seizure }\end{array}$ & Number & $\begin{array}{l}\text { Sleep/ } \\
\text { Awake }\end{array}$ & $\begin{array}{l}\text { Free } \\
(y)\end{array}$ & Ictal symptoms in sequence & $\begin{array}{l}\text { Duration } \\
\text { (mins) }\end{array}$ & \\
\hline $1 \mathrm{M}$ & 14 & 8.5 & 8.5 & 1 & Sleep & 5.5 & V-LOC-stiffness & 5 & Right central spikes and GSSES \\
\hline $2 \mathrm{M}$ & 16 & 5.5 & 6 & 2 & Sleep & 10 & LHC-V-LOC-flaccid-IU & 30 & Right central spikes and GSSES \\
\hline $3 \mathrm{~F}$ & 13 & 4 & 4.5 & 2 & Sleep & 8.5 & LHC-OPS-V-salivation-speech arrest & 10 & Midline $(\mathrm{Pz})$ spikes \\
\hline $4 \mathrm{M}$ & 19 & 5 & 10 & 50 & Sleep & 9 & ED-V-LOC-LHC, postictal HP & 4 & Midline $(\mathrm{Cz}$ and $\mathrm{Pz})$ spikes \\
\hline $5 \mathrm{M}$ & 14 & 5 & 6 & 3 & Sleep & 9 & ED-V-speech arrest & 15 & Left frontal spikes \\
\hline $6 \mathrm{M}$ & 6 & 3.5 & 3.5 & 1 & Sleep & 2.5 & V-ED-LOC-GC status & 5 & Photoparoxysmal spike-slow wave \\
\hline $7 \mathrm{M}$ & 6 & 2 & 4 & 3 & Sleep & 2 & ED-V-LOC-RHC, postictal HP & 5 & Normal \\
\hline $8 \mathrm{M}$ & 23 & 6 & 6 & 1 & Awake & 17 & Dizziness-speech arrest-V-P-GTCS & 5 & Normal \\
\hline $9 M$ & 15 & 11 & 11 & 1 & Sleep & 4 & V-LOC-RHC & 30 & Normal \\
\hline Mean & 14 & 5.6 & 6.6 & 7.1 & & 7.4 & & 12.1 & \\
\hline $\mathrm{SD}$ & 5.5 & 2.7 & 2.7 & 16.1 & & 4.6 & & 10.7 & \\
\hline Median & 14 & 5 & 6 & 2 & & 8 & & 5 & \\
\hline Maximum & 23 & 11 & 11 & 50 & & 17 & & 30 & \\
\hline Minimum & 6 & 2 & 4 & 1 & & 2 & & 4 & \\
\hline
\end{tabular}

FU=Follow-up; V=vomiting; LOC=loss of consciousness; LHC or RHC=left (L) or right (R) sided clonic convulsions; IU=incontinence of urine, ED=deviation of the eyes, $\mathrm{P}=$ pallor; GSStatus=generalised convulsive status epilepticus; OPS=oropharyngolaryngeal symptoms; HP=hemiparesis; GSSES=giant somatosensory evoked spikes.

\section{Results}

PREVALENCE, CHRONOLOGICAL, AND CLINICAL

DATA

Prevalence

These nine children constitute $9.2 \%$ of 98 patients with BCPS (94 had EEG spike foci) or $2.2 \%$ of 418 patients with onset of seizures before the age of 13 years.

Sex, age, and other chronological data are shown in the table. Eight were boys.

Neurological and mental state, and development All patients were normal and attended main stream school often with good performance. Patient 2 was mildly hyperkinetic with low grade school records. Patient 4 was considered as badly behaving. Only one child had mild headaches.

Family history

There was no other member of the family with similar seizures. Patient 6 had a strong family history of febrile convulsions.

Clinical data

All patients had seizures with ictal vomiting either from the onset or during the ictus (table). In all but one patient fits occurred during sleep. All but one (patient 4) had single or a maximum of three fits (table). There was no postictal headache after partial seizures.

Description of seizures

Patient 1 was found vomiting with loss of consciousness and generalised stiffness.

Patient 2 had left arm clonic convulsions with concurrent vomiting for 30 minutes, then becoming flaccid, unresponsive, and incontinent of urine for another 20 minutes before gradual recovery. A shorter similar seizure occurred 1 year later.

Patient 3 had simple partial left sided clonic convulsions associated with oropharyngolaryngeal symptoms, vomiting, hypersalivation, and speech arrest.

Patient 4 had frequent seizures of eyes deviated to the left with vomiting, progressing to left hemiconvulsions and mild postictal hemiparesis. Seizure control was achieved with treatment.
Patient 5 had, within 2 weeks, two seizures witnessed from the onset. These consisted of tonic deviation of the eyes to the right with vomiting. He could understand but was unable to speak. He had a further similar seizure 1 year later despite treatment with $200 \mathrm{mg}$ carbamazepine daily.

Patient 6 was treated with phenobarbitone because of three febrile convulsions. Three days after sudden drug withdrawal, a seizure occurred with vomiting, deviation of the eyes, and unresponsiveness followed within 5 minutes by generalised convulsive status epilepticus.

Patient 7 had eyes deviated to the right, vomiting, and loss of consciousness followed within 5 minutes by prolonged right hemiconvulsions and postictal hemiparesis. A similar but milder seizure with the same sequence of events and brief hemiconvulsions occurred 2 months later. Despite receiving phenobarbitone, another seizure was witnessed from the beginning. He sat on his bed; his eyes opened and immediately deviated to the right. Within seconds he started vomiting and became unresponsive for 5 minutes without convulsions.

Patient 8 had a diurnal simple partial seizure that started with dizziness, speech arrest, vomiting, and pallor for 5 minutes, ending with generalised convulsions.

Patient 9 had vomiting and unresponsiveness for 30 minutes before progressing to hemiconvulsions.

\section{ELECTROENCEPHALOGRAPHY}

All patients had one to three EEGs in the first year after their first seizure and annual EEGs in the next 3-7 years. The EEG spike foci were seen only once and in their first EEG (patients 3 and 4) or persisted for 1-2 years (patients 1 and 2). Patient 5 had a normal first EEG but all subsequent EEGs consistently showed a high amplitude spike and slow wave focus in the left frontal electrode, before complete normalisation at the age of 9 . All spike foci, in all patients, were of high amplitude and morphologically similar to the centrotemporal or occipital paroxysms of the BCPS (figure). Patient 6 had a normal EEG at the age of 2 years but another EEG 1 year later showed a brief generalised 
A

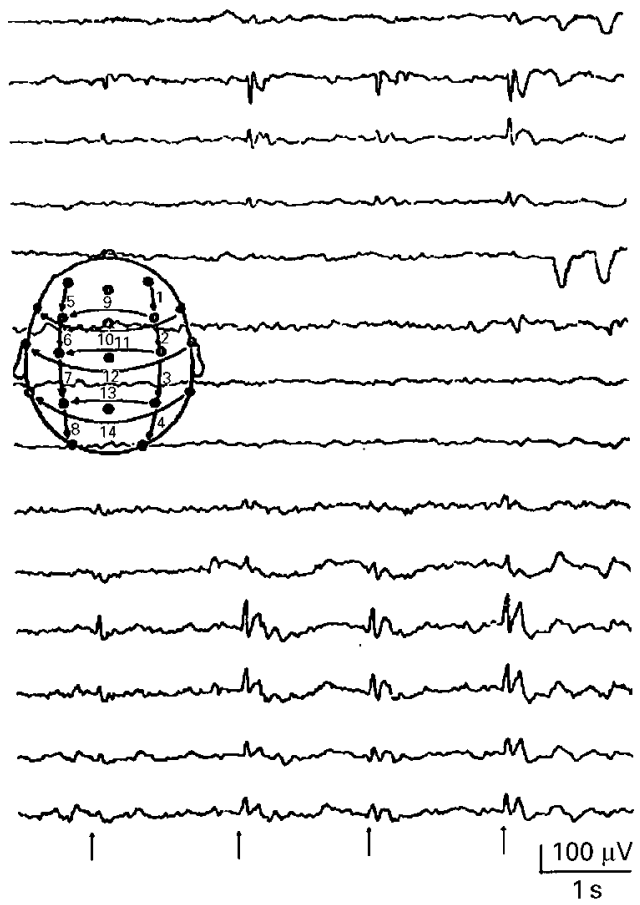

$B$

F8-F4

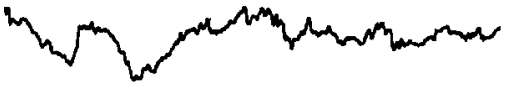

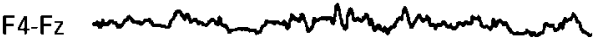
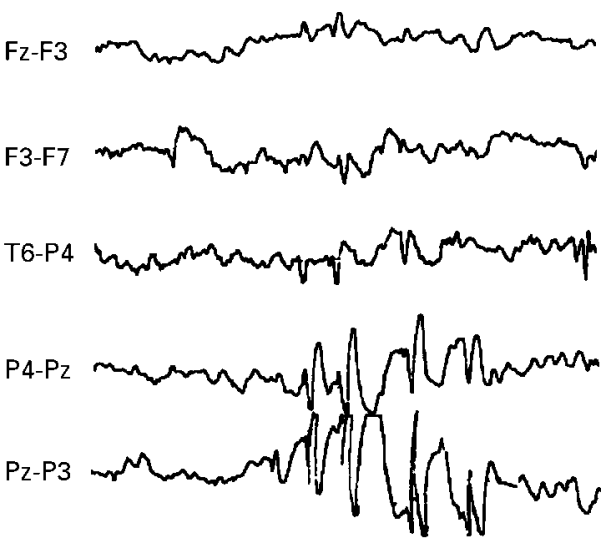

P3-T5

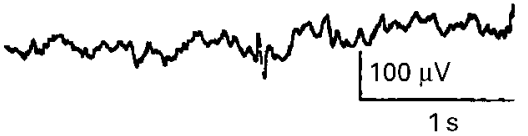

(A) EEG of patient 1 with right sided spontaneous central spikes that were also consistently elicited by tapping (arrows) the fingers of the left hand. (B) EEG of patient 3 with high amplitude spikes localised in the posterior midline (Pz) electrode.

spike and slow wave on photic stimulation that was not seen in subsequent EEG.

TREATMENT AND PROGNOSIS

Patients 1 and 9 were not treated. Patients 2, 5, and 8 received carbamazepine and patients 3 , 4,6 , and 7 phenobarbitone or cyclohexyl-2methylamino-propranol-phenylethyl barbiturate for 2-6 years. All patients did well with no further seizures and good development (table).

\section{Discussion}

Ictal vomiting, the shared seizure symptom of these patients, occurs mainly in children with spontaneous ${ }^{1-6}{ }^{8-9}$ or photically induced occipital seizures. ${ }^{8}$ Before our publication, ${ }^{11}$ ictal vomiting was reported in only 16 cases according to a recent report of two additional patients. ${ }^{10}$ The consensus from ictal recordings of mainly symptomatic epilepsies is that ictal vomiting is activated from the non-dominant temporal lobe and insular. ${ }^{10}$ Although this may be true in symptomatic patients, it does not explain its scarcity in adults with most of the reported patients showing bilateral epileptogenic foci, ${ }^{10}$ ictal EEG with mainly left sided abnormalities $^{3}$ and that children, particularly with benign idiopathic spontaneous ${ }^{13-6}$ or photically induced occipital seizures, ${ }^{8}$ are far more susceptible than adults. ${ }^{11}$ Ictal vomiting may require more than one contributing factor. ${ }^{11}$

Irrespective of pathogenetic mechanisms, these nine children with partial seizures and ictal vomiting have an entirely benign condition documented with long follow up. In this respect they classify amongst the BCPS that have two main representatives, benign childhood seizures with centrotemporal spikes that are well known ${ }^{712}$ and EBOS described in the introduction. ${ }^{1-6}$ Benign childhood partial seizures may also manifest with visual fits and occipital paroxysms, ${ }^{72}$ affective ictal symptomatology and frontotemporoparietal spikes, ${ }^{13}$ infrequent, diurnal versive seizures of the head and body often without impairment of consciousness, and giant somatosensory evoked spikes ${ }^{14}$ or photically induced occipital seizures often with ictal vomiting. ${ }^{8}$

These BCPS share common clinical and EEG characteristics. ${ }^{7}$ Seizures are infrequent, usually nocturnal, and remit within $1-3$ years from onset. Brief or prolonged fits, even status epilepticus, may be a singular ictal event in a lifetime. Ictal hypersalivation, vomiting, pallor, or sweating, unusual in other epileptic syndromes, are frequent and may occasionally appear in isolation. Children with the clinical and EEG characteristics of one form may evolve into or simultaneously develop features of another form of BCPS. Febrile convulsions are common. Neurological examination, intellect, and brain imaging are normal. High amplitude unilateral or bilateral giant sharpslow wave foci on EEC are usually abundant and disproportionately severe to seizure infrequency. They may be highly localised or combined with foci from other cortical areas or with brief generalised discharges and exaggerate with sleep. Similar EEG features resolving with age are found in $2 \%-4 \%$ of normal children, and more often in children having an EEG for reasons other than seizures.

Because of the above shared characteristics, a unified concept of BCPS has been proposed. ${ }^{7}$ The term benign childhood seizure susceptibility syndrome or benign childhood partial seizures is used to denote exactly what 
these children have-that is, an idiopathic propensity to partial seizures, EEG functional focal spikes or both which are age dependent and age limited. Benign childhood seizure susceptibility is a common, probably genetically determined, mild and reversible, functional derangement of the brain cortical maturational process. ${ }^{1516}$ This is often clinically silent manifesting in more than $90 \%$ with EEG sharp and slow waves only. ${ }^{16}$ The others may have infrequent partial seizures. EEG and seizure manifestations often follow an age related localisation and prevalence. Thus, this cortical excitability is expressed from the occipital cortex of younger children at a peak age of onset of 5 years when the centrogyral and neighbouring cortex becomes active at a later age at 7-9 years. ${ }^{16}$ Centrogyral location is 2.5 times more frequent than occipital location, and other cortical areas are less often actively involved. ${ }^{16}$

The nine children of this report signify with their symptoms and EEG this unified concept of a benign childhood partial seizure susceptibility syndrome. They all have ictal vomiting that is a cardinal symptom of EBOS, yet none of them had occipital spikes in their EEG. Conversely, five of them had central, giant somatosensory, frontal or midline spikes of other BCPS that do not manifest with ictal vomiting. Furthermore, some of these patients also have, concurrently with ictal vomiting, other manifestations such as hemiclonic seizures, oropharyngolaryngeal symptoms, speech arrest, and hypersalivation that characterise other than the occipital BCPS. Irrespective of their categorisation, their combined clinico-EEG manifestations are unlikely to occur in other than benign childhood partial epilepsies and in this respect are important to recognise for prognostic purposes. Most of these children may not need treatment.

1 Panayiotopoulos CP. Benign childhood epilepsy with Panayiotopoulos CP. Benign childhood epilepsy with
occipital paroxysms: a 15 -year prospective study. Ann Neuoccipital paroxysm

2 Kivity S, Lerman P. Stormy onset with prolonged loss of consciousness in benign childhood epilepsy with occipital paroxysms. F Neurol Neurosurg Psychiatry 1992;55:45-8.

3 Vigevano F, Ricci S. Benign occipital epilepsy of childhood with prolonged seizures and autonomic symptoms. In: Andermann F, Beaumanoir A, Mira L, et al, eds. Occipital seizures and epilepsies in children. London: John Libbey, 1993:133-40.

4 Maher J, Ronen GM, Ogunyemi AO, et al. Occipital paroxysmal discharges suppressed by eye opening: variability in clinical and seizure manifestations in childhood. Epilepsia 1995;36:52-7.

5 Fejerman N. Atypical evolutions of benign partial epilepsies in children. International Pediatrics 1996;11:351-6.

6 Ferrie CD, Beaumanoir A, Guerrini R, et al. Early-onset benign occipital seizure susceptibility syndrome. Epilepsia 1997;38:285-93.

7 Panayiotopoulos CP. Benign childhood partial epilepsies: benign childhood seizure susceptibility syndromes [editobenign childhood seizure susceptibility syndrom
rial]. F Neurol Neurosurg Psychiatry 1993;56:2-5.

8 Gial]. F Neurol Neurosurg Psychiatry 1993;56:2-5. Guerrini R, Dravet C, Genton P, et al. Idiopathic photosen-
sitive occipital lobe epilepsy. Epilepsia 1995;36:883-91.

9 Guerrini R, Belmonte A, Veggiotti P, et al. Delayed appearance of interictal EEG abnormalities in early onset childhood epilepsy with occipital paroxysms. Brain Dev 1997;19:343-6.

10 Devinsky O, Frasca J, Pacia SV, et al. Ictus emeticus: further evidence of nondominant temporal involvement. Neurology 1995;45:1158-60.

11 Panayiotopoulos CP. Vomiting as an ictal manifestation of epileptic seizures and syndromes. 7 Neurol Neurosurg Psychiatry 1988;51:1448-51.

12 Commission on Classification and Terminology of the International League Against Epilepsy. Proposal for revised classification of epilepsies and epileptic syndromes. Epilepsia 1989;30:389-99.

13 Dalla Bernardina B, Bureau M, Dravet C, et al. Affective symptoms during attacks of epilepsy in children. Revue $d$ symptoms during attacks of epilepsy in children. Revue d
Electroencephalographie et de Neurophysiologie Clinique 1980; Electroenceph 18 .

14 Tassinari CA, De Marco P. Benign partial epilepsy with extreme somato-sensory evoked potentials.In: Roger J, Bureau M, Dravet C, et al, eds. Epileptic syndromes in infancy, childhood and adolescence. London: John Libbey, 1992: 225-9.

5 Sorel L, Rucquoy-Ponsar M. L'epilepsie functionelle de maturation. Apport des montages verticaux en E.E.G. dans le diagnostic de cette forme d'epilepsie. Revue Neurologique 1969;121:289-297.

16 Panayiotopoulos CP. Benign childhood seizures and related epileptic syndromes. London: John Libbey \& Company Ltd, 1998 (in press) 